\title{
ON THE FREDHOLM INTEGRAL EQUATION ASSOCIATED WITH PAIRS OF DUAL INTEGRAL EQUATIONS
}

\author{
by V. HUTSON
}

(Received 2nd December 1967)

1. Consider the Fredholm equation of the second kind

where

$$
f(x)-\int_{0}^{1} k_{a}(x, y) f(y) d y=h(x) \quad(0 \leqq x \leqq 1),
$$

$$
k_{a}(x ; y)=\int_{0}^{\infty} k_{a}(t)(x t)^{\frac{1}{2}} J_{v}(x t)(y t)^{\frac{1}{2}} J_{v}(y t) d t \quad\left(v \geqq-\frac{1}{2}\right),
$$

and $J_{v}$ is the Bessel function of the first kind. Here $k_{a}(t)$ and $h(x)$ are given, the unknown function is $f(x)$, and the solution is required for large values of the real parameter $a$. Under reasonable conditions the solution of (1.1) is given by its Neumann series (a set of sufficient conditions on $k_{a}(t)$ for the convergence of this series is given in Section 4, Lemma 2). However, in many applications the convergence of the series becomes too slow as $a \rightarrow \infty$ for any useful results to be obtained from it, and it may even happen that $f(x) \rightarrow \infty$ as $a \rightarrow \infty$. It is the aim of the present investigation to consider this case, and to show how under fairly general conditions on $k_{a}(t)$ an approximate solution may be obtained for large $a$, the approximation being valid in the norm of $L_{2}(0,1)$. The exact conditions on $k_{a}(t)$ and the main result are given in Section 4. Roughly, it is required that $1-k_{a}(a t)$ should behave like $t^{\rho}(\rho>0)$ as $t \rightarrow 0$. For example, $k_{a}(t)$ might be $\exp \left[-(t / a)^{p}\right]$.

Equation (1.1) occurs in the solution of a class of mixed boundary value problems. This type of problem often leads to the following pair of dual integral equations:

$$
\begin{aligned}
\int_{0}^{\infty} t^{-2 \alpha}\left[1-k_{a}(t)\right] L(t) J_{v+\alpha}(x t) d t & =M(x) & & (0 \leqq x \leqq 1), \\
\int_{0}^{\infty} L(t) J_{v+\alpha}(x t) d t & =0 & & (x>1),
\end{aligned}
$$

and it is well known that these equations may be reduced formally to (1.1); this reduction is considered in detail in (9). The rigorous analysis of (1.1) is more straightforward than that of (1.3) and (1.4), and this investigation will be mainly concerned with (1.1). However, in Section 5 some results are obtained for the approximate solution of dual integral equations. These results are relevant to the discussion in Sneddon (9), Section 4.8.

$$
\text { E.M.S.-M }
$$


Examples of cases where (1.1) occurs are problems considered by Love (7), Cooke (2) and Mahalanabis (8). In (5) the author has used a particular case of the method in obtaining an approximate solution for the coaxial disc condenser at small separations.

An outline of this investigation is as follows. In Sections 2 and 3 the problem is formulated in the Hilbert space $L_{2}(0,1)$ and the approximate solution is obtained in an abstract form. The results in these sections are not new and are a generalisation by the author (4) of results of Widom (10) and (11) for translation kernels. In Section 4 the explicit approximate solution of (1.1) is given. In Section 5 the approximate solution of a pair of dual integral equations is discussed.

2. In this section some of the standard properties of the Hankel transform are given. A family of operators $A_{a}$ depending on a parameter $a$ will then be defined in terms of the transform, and the approximate inversion of $A_{a}$ will be discussed formally. It will be shown in Section 4 that the integral equation (1.1) may be studied by considering operators of this kind.

Denote by $L_{2}(0, \infty)$ the Hilbert space of complex valued, measurable, square integrable functions defined on $[0, \infty)$ with the usual inner product $(f, g)$ and norm $\|f\| . L_{2}(0,1)$ will denote the closed subspace of $L_{2}(0, \infty)$ composed of functions vanishing outside $[0,1]$. The Hankel transform is a particular case of the general transforms studied in (1), and its definition and relevant properties are given in the following theorems.

Theorem 1. For any $f, g \in L_{2}(0, \infty)$ and any $v \geqq-\frac{1}{2}$ the limit

$$
\hat{f}(t)=H_{v}(f)=\lim _{A \rightarrow \infty} \int_{0}^{A}(x t)^{\frac{1}{2}} J_{v}(x t) f(x) d x
$$

exists in the norm of $L_{2}(0, \infty)$ and the mapping $f \rightarrow \hat{f}$, called the Hankel transform, is a self inverse isometric isomorphism of $L_{2}(0, \infty)$ onto itself, so that

$$
(\hat{f}, \hat{g})=(f, g) \text {. }
$$

Theorem 2. Suppose $f, g \in L_{p}(0, \infty)$ for some $p, 1<p \leqq 2$. Then for any $v \geqq-\frac{1}{2}$ the limit defined in $(2.1)$ exists in the norm of $L_{q}(0, \infty)$ where $p^{-1}+q^{-1}=1$. Also the following relation holds

$$
\int_{0}^{\infty} f(t) \hat{g}(t) d t=\int_{0}^{\infty} f(t) g(t) d t
$$

Denote by $\hat{L}_{2}(0,1)$ the image of $L_{2}(0,1)$ under the Hankel transform. The domain and range of an operator, say $A$, will be written $\mathfrak{D}(A)$ and $\mathfrak{R}(A)$ respectively.

Let $P(t)$ be a real, measurable and uniformly bounded function. Then clearly the equation

$$
(A f, g)=(P(t) \hat{f}(t), \hat{g}(t)) \quad\left(f, g \in L_{2}(0,1)\right),
$$


defines a linear, continuous, self adjoint operator on $L_{2}(0,1)$ into itself. It will be shown in the next section that even if $P(t)$ is not bounded it is often still possible to define an unbounded self adjoint operator $A$ corresponding to $P$ so that (2.4) holds for $f$ and $g$ in a dense subset of $L_{2}(0,1)$.

Suppose now that $P_{a}(t)$ is a family of functions depending on a parameter $a$, and $A_{a}$ is the corresponding family of operators. If as $a \rightarrow \infty, P_{a}(t) \rightarrow P(t)$ for every $t$, then it might be expected that $A_{a}$ would tend to $A$ in some sense. For the cases of interest this convergence only holds in a certain generalised sense, see (6). However, it will be shown in the next section that under reasonable conditions on $P_{a}$ and $P$ the operators all have continuous inverses, and as $a \rightarrow \infty, A_{a}^{-1} \rightarrow A^{-1}$ in the uniform operator norm of $L_{2}(0,1)$. This property gives the approximate inverse of $A_{a}$ for large $a$, and will be used to find the approximate solution of (1.1).

3. The discussion in this section is based on results obtained by Widom (10) for the Fourier transform and extended by the author (4) to more general transforms of which the Hankel transform is a special case.

Let $P(t)$ be a real, non-negative measurable function defined on $[0, \infty)$ which vanishes on a set of measure zero. Suppose also that $P(t)$ is essentially bounded on every compact set and that it is bounded by a polynomial as $t$ approaches infinity. Let $\hat{H}$ be the set of functions in $\hat{L}_{2}(0,1)$ such that

With the inner product

$$
\int_{0}^{\infty}[1+P(t)]|\hat{f}(t)|^{2} d t<\infty
$$

$$
[\hat{f}, \hat{g}]=\int_{0}^{\infty}[1+P(t)] \hat{f}(t) \hat{\bar{g}}(t) d t
$$

and norm $\|[\hat{f}]\|=[\hat{f}, \hat{f}]^{\frac{1}{2}}, \hat{H}$ is clearly a Hilbert space, and it is shown in (4) that the set $\hat{H}$ is dense in $\hat{L}_{2}(0,1)$. Also

$$
\|[\hat{f}]\|>\|f\| \quad(\hat{f} \neq 0) \text {. }
$$

The relation $[\hat{f}, \hat{g}]=[f, g]$ defines another Hilbert space $H \subset L_{2}(0,1)$.

$H$ will be used to define an operator $A$, unbounded in general, by means of the following result easily deduced from (6, VI Theorems (2.1), (2.6)).

Lemma 1. There exists a unique, linear, self adjoint (densely defined) operator $A$ on $L_{2}(0,1)$ into itself with $\mathfrak{D}(A) \subset H$ such that

$$
((I+A) f, g)=[f, g]=[\hat{f}, \hat{g}] \quad(f \in \mathfrak{D}(A), g \in H) .
$$

$A$ is positive in the sense that

$$
(A f, f)>0 \quad(f \in \mathfrak{D}(A), f \neq 0) .
$$

Further properties of $A$ are obtained by using (i) and (ii) below which follow immediately from the definition of the Hankel transform.

(i) If $f^{(n)} \in L_{2}(0,1)$ and $f^{(n)}$ converges weakly in $L_{2}(0,1)$ to $f$, then $f^{(n)}(t)$ converges pointwise to $\hat{f}(t)$. 
(ii) Let $U=\left\{f \mid f \in L_{2}(0,1),\|f\| \leqq 1\right\}$. Then for any given finite subinterval $E$ of $[0, \infty)$ there exists $\hat{g}_{E}(t)$ in $L_{2}(0, \infty)$ such that for all $f \in U, t \in E$, $|\vec{f}(t)|<\hat{g}_{E}(t)$. Under these conditions it was shown in (4) Theorem 2, Corollary 2 that the following result holds.

Theorem 3. Let $L=\operatorname{ess}\lfloor(t)$ as $t \rightarrow \infty$. The spectrum of $A$ to the left of $L$ consists of strictly positive eigenvalues of finite multiplicity whose only possible limit point is $L$. If $L=\infty$ then $A^{-1}$ is a positive definite compact operator.

Corollary. Let $L=\infty$. If $R$ is a bounded operator on $L_{2}(0,1)$ such that $\Re(R) \subset H$ and

$$
[R f, g]=((I+R) f, g)
$$

for all $f$ and all $g \in H$, then $A^{-1}=R$.

Proof. By Theorem $3, A^{-1}$ is compact. Therefore, for any $f$ there exists an $h \in \mathfrak{D}(A)$ with $f=A h$. Hence from (3.4) and (3.6)

$$
[(R A-I) h, g]=((R A-I) h, g) \text {. }
$$

Now set $g=(R A-I) h$. Then from (3.3), $(R A-I) h=0$, and so $R f=h=A^{-1} f$ for all $f$. This completes the proof.

Now consider a family of functions $P_{a}(t)$ depending on the parameter $a$, each of which is defined in the same manner as $P(t)$. Let $A_{a}$ be the corresponding family of operators defined by Lemma 1 . Suppose the following conditions hold.

(3.7) As $a \rightarrow \infty, P_{a}(t) \rightarrow P(t)$ pointwise.

(3.8) There exist numbers $a_{0}, c_{1}, c_{2}$ such that $P_{a}(t) \leqq c_{1}+c_{2} P(t)$ for all $a>a_{0}$ and all $t$.

(3.9) Given any $\varepsilon>0$, there exist numbers $t_{0}, a_{0}$ such that for $a>a_{0}$, $t>t_{0}, P_{a}(t) \geqq \varepsilon^{-1}$.

The convergence of $A_{a}$ to $A$ is given by the following result.

Theorem 4. If (3.7), (3.8) and (3.9) are satisfied, then for a large enough $A_{a}^{-1}$ is continuous. If $L=\infty$, then as $a \rightarrow \infty, A_{a}^{-1} \rightarrow A^{-1}$ in the uniform operator norm of $L_{2}(0,1)$.

Proof. From (3.9) for $a$ large enough, as $t \rightarrow \infty$, $\varliminf_{a}(t)>0$. Therefore by Theorem 3 zero is not in the spectrum of $\boldsymbol{A}_{a}$, so that $A_{a}^{-1}$ is continuous. The remainder of the result is proved in (4) Theorem 2, Corollary 2.

4. The results of Sections 2 and 3 will now be used to investigate the integral equation (1.1). Lemma 2 below gives a sufficient condition for the convergence of the Neumann series (a remark on this point is made in (3)). However, the main result, Theorem 5 below, deals with the case when this series converges more and more slowly as $a \rightarrow \infty$ and shows that it is still possible to obtain an approximate solution for a rather large class of kernel.

Suppose the real valued function $k_{a}(t)$ defined for $t \geqq 0$ and $a>a_{1}$ satisfies the following conditions: 
(4.1) For each $a, k_{a}(t)$ is either in $L_{1}(0, \infty)$ or $L_{2}(0, \infty)$.

(4.2) $k_{a}(0)=1$ and for $t>0, k_{a}(t) \geqq-1+\delta$ for some $\delta>0$. Also given any $\delta>0$, there exist numbers $a_{0}$ and $l$ such that

$$
k_{a}(a t) \leqq l<1 \quad\left(t \geqq \delta, a \geqq a_{0}\right) .
$$

(4.3) There exist positive numbers $b, \rho$ such that

$$
b^{-1} t^{-\rho}\left[k_{a}(a t)-1+b t^{\rho}\right]
$$

approaches zero uniformly (with respect to $a$ ) as $t \rightarrow 0$.

Let $K_{a}$ be the integral operator on $L_{2}(0,1)$ with kernel $k_{a}(x, y)$ defined by (1.2), so that

$$
\left(K_{a} f\right)(x)=\int_{0}^{1} k_{a}(x, y) f(y) d y \quad\left(f \in L_{2}(0,1)\right) .
$$

The integral equation (1.1) may then be written

$$
\left(I-K_{a}\right) f=h \quad\left(h \in L_{2}(0,1)\right) .
$$

By using the Hankel transform together with (2.2), it follows that

$$
\left(\left(I-K_{a}\right) f, g\right)=\left(\left(1-k_{a}(t)\right) \hat{f}(t), \hat{g}(t)\right) \quad\left(f, g \in L_{2}(0,1)\right),
$$

where condition (4.1) has been used. Now set

Then from (3.4)

$$
P_{a}(t)=b^{-1} a^{\rho}\left(1-k_{a}(t)\right) .
$$

$$
A_{a}=b^{-1} a^{\rho}\left(I-K_{a}\right)
$$

We then have the following result.

Lemma 2. Let $a$ be fixed. Suppose that either $k_{a}(t) \in L_{1}(0, \infty)$ or $k_{a}(t) \in L_{2}(0, \infty)$. Suppose that for each $\delta>0$ there exists $l<1$ with $k_{a}(t) \leqq l(t>\delta)$, and for some $\gamma>0, k_{a}(t) \geqq-1+\gamma(t \geqq 0)$. Then the Neumann series of $(4.5)$ is convergent in the norm of $L_{2}(0,1)$ so that

$$
f=\sum_{n=0}^{\infty} K_{a}^{n} f\left(h \in L_{2}(0,1)\right) .
$$

Proof. It follows from (4.7) that $\underline{\lim } P_{a}(t)>0$. By Theorem 3 the spectrum of $A_{a}$ is to the right of some $\varepsilon>0$. Therefore the spectrum of $K_{a}$ is by (4.8) to the left of $1-b a^{-\rho_{\varepsilon}}$. Also $k_{a}(t) \geqq-1+\gamma$ so the spectrum of $K_{a}$ is to the right of $-1+\gamma$. Hence the spectral radius of $K_{a}$ is less than unity. The result follows immediately.

If (4.3) holds the series (4.9) will converge more and more slowly as $a \rightarrow \infty$ and so will be of little use in finding a solution of (4.5) for large $a$. In this case an approximate solution is given by Theorem 5 below.

Let $R$ be the integral operator on $L_{2}(0,1)$ with kernel $R(x, y)$; that is,

$$
(R h)(x)=\int_{0}^{1} R(x, y) h(y) d y,
$$


$R(x, y)=2^{2-\rho}\left[\Gamma\left(\frac{\rho}{2}\right)\right]^{-2}(x y)^{v+\frac{1}{2}} \int_{\max (x, y)}^{1} u^{-2 v-\rho+1}\left[\left(u^{2}-x^{2}\right)\left(u^{2}-y^{2}\right)\right]^{\frac{1}{2 \rho-1}} d u$,

where $R$ is defined for $0 \leqq x, y \leqq 1$ and $x \neq y$.

Theorem 5. Suppose (4.1), (4.2) and (4.3) are satisfied and $v \geqq-\frac{1}{2}$. Then if $\|h\| \neq 0$, the integral equation (4.5) has a unique solution $f \in L_{2}(0,1)$ and

$$
f=b^{-1} a^{\rho}\left(R h+\delta_{a}\right) \text {. }
$$

We have $\|R h\| \neq 0$ and $\left\|\delta_{a}\right\| \leqq \varepsilon_{a}\|h\|$, where $\varepsilon_{a}$ is a set of numbers independent of $h$ and $\varepsilon_{a} \rightarrow 0$ as $a \rightarrow \infty$.

Proof. It is a simple matter to check that with $P(t)=t^{\rho}$ and $P_{a}(t)$ given by (4.7) conditions (3.7), (3.8) and (3.9) are satisfied. Evidently $L=\infty$, so that by Theorem $4, A_{a}^{-1} \rightarrow A^{-1}$ uniformly. It is proved in the appendix that $A^{-1}=R$. The result follows on using (4.8).

It will usually be the case that this approximation may be iterated by substituting the approximate solution in (4.5) and using (4.12) again. For this to be valid it is only necessary that the new right hand side of (4.5) should be small in norm as $a \rightarrow \infty$. An example where an iteration has been used is given in (5).

5. Consider the dual integral equations

$$
\begin{gathered}
\int_{0}^{\infty} P_{a}(t) \hat{f}(t)(x t)^{\frac{1}{2}} J_{v}(x t) d t=h(x) \quad(0 \leqq x \leqq 1), \\
\int_{0}^{\infty} \hat{f}(t)(x t)^{\frac{1}{2}} J_{v}(x t) d t=0 \quad(x>1),
\end{gathered}
$$

where $P_{a}(t)$ satisfies conditions (3.7), (3.8) and (3.9) with $P(t)=t^{\rho}(\rho>0)$.

These equations are easily reduced by a formal argument to

$$
\left(P_{a}(t) \hat{f}(t), \hat{g}(t)\right)=(h, g) \quad(f, g \in H) .
$$

It follows from Lemma 1 and Theorem 4 that, for any $h \in L_{2}(0,1), f=A_{a}^{-1} h$. If for each $a, P_{a}(t)$ is uniformly bounded then $\hat{f}$ will be the solution of (5.1) and (5.2), and Theorem 4 will give an approximate solution of these equations. However this is not true for general $P_{a}(t)$. For example suppose $a$ is fixed and $P_{a}(t)=t^{2 n}$ where $n$ is a positive integer. Then it is easy to see from (A5) that there will always be a function $h$ in $L_{2}(0,1)$ such that $t^{n} \hat{f}(t)$ is in $L_{2}(0, \infty)$ but $t^{2 n} f(t)$ is not in $L_{p}(0, \infty)$ for any $p \geqq 1$, so that the left hand side of $(5.1)$ cannot be interpreted as a transform. In this case $f$ is a weak solution in the sense that (5.3) is satisfied. The following result now holds, $R$ being given by (4.10) and (4.11).

Theorem 6. Let $h \in L_{2}(0,1)$ and suppose that one of the following conditions holds.
(i) $P_{a}(t) \leqq M_{a}(t \geqq 0)$ for some set $M_{a}$ of finite numbers.
(ii) $\left(1+t^{\rho}\right)(\widehat{R h})(t) \in L_{2}(0, \infty)$. 
Then the solution of (5.1) and (5.2) is given by

$$
\hat{f}=b^{-1} a \rho\left[R h+\delta_{a}\right]
$$

where $\left\|\delta_{a}\right\| \leqq \varepsilon_{a}\|h\|$ and $\varepsilon_{a} \rightarrow 0$ as $a \rightarrow \infty$ with $\varepsilon_{a}$ independent of $h$.

Appendix. The operator $A^{-1}$ corresponding to $P(t)=t^{\rho}$ will now be obtained. This may be done by a formal argument from the dual integral equations (5.1) and (5.2). However, as remarked in Section 5, the " solution" obtained does not in general satisfy the original equations in any ordinary sense. These equations will not therefore be used in the proof. The method adopted will be to show that (5.3) is satisfied. This is analogous to the method used by Widom (10) for translation kernels.

It is convenient in the subsequent analysis to use the notation $H_{v}(f)$ for the Hankel transform of $f$. As before $L_{2}(0,1)$ is the set of functions in $L_{2}(0, \infty)$ with support in $[0,1]$.

Theorem 7. If $P(t)=t^{\rho}(\rho>0)$ and $v \geqq-\frac{1}{2}$, then $A^{-1}=R$ where $R$ is given by (4.10) and (4.11).

Proof. The proof utilises the Corollary to Theorem 3. It is simple to show by estimating approximately the integral in (4.11) that for each $\rho>0$ there is a constant $M_{\rho}$ such that

$$
|R(x, y)| \leqq M_{\rho}\left\{1+|x-y|^{\rho-1}+\log |x-y|^{-1}\right\} .
$$

It follows from (6) Chapter III, 2.4 that $R$ is a bounded operator on $L_{2}(0,1)$. It will be proved that $\mathfrak{R}(R) \subset H$. Also it will be shown that

$$
\left(t^{\frac{1}{\rho} \rho} H_{v}(R f), t^{\frac{1}{2} \rho} H_{v}(g)\right)=\left(H_{v}(f), H_{v}(g)\right) \quad(g \in H),
$$

which is equivalent to (3.6). By the Corollary to Theorem 3, this shows that $A^{-1}=R$.

Let $\rho=2 n+\beta$, where $n$ is an integer and $0 \leqq \beta<2$. The following relations hold.

$$
\begin{gathered}
t^{2 n} H_{v}(R(x, y))=(y t)^{\frac{1}{1}} J_{v}(y t)-y^{v+\frac{1}{2}} \sum_{r=0}^{n-1} b_{r} t^{r+\frac{1}{t}}\left(1-y^{2}\right)^{r} J_{v+r}(t) \quad(\rho=2 n), \\
t^{\rho} H_{v}(R(x, y))=(y t)^{\frac{1}{ \pm}} J_{v}(y t)-y^{v+\frac{1}{2}}\left(1-y^{2}\right)^{\frac{1}{2} \rho} \sum_{r=0}^{n} a_{r, \rho} t^{r+\frac{1}{2}} \\
\times \int_{1}^{\infty} \frac{z^{-v-r+1} J_{v+r}(z t)}{\left(z^{2}-y^{2}\right)^{n-r+1}\left(z^{2}-1\right)^{-n+\frac{1}{t} \rho}} d z \quad(\rho>0),
\end{gathered}
$$

where $a_{r, \rho}$ and $b_{r}$ are constants that depend only on $r, \rho$ and $r$ respectively. These relations may be proved by a straightforward but tedious calculation using standard Bessel function identities, and their proof will be omitted. The following Lemmas will be used in the proof.

Lemma A1. Let $f \in L_{2}(0,1)$. Define $F_{r, \rho}(z)=0$ for $0 \leqq z \leqq 1$, and for $z>1$ set

$$
F_{r, p}(z)=\left(z^{2}-1\right)^{-\frac{1}{t \beta} z^{-v-r+\frac{1}{2}}} \int_{0}^{1} y^{v+\frac{1}{2}}\left(1-y^{2}\right)^{n+\frac{1}{2} \beta}\left(z^{2}-y^{2}\right)^{-n+r-1} f(y) d y,
$$


where $r$ is an integer with $0 \leqq r \leqq n$. Then $F_{r, \rho}(z) \in L_{p}(0, \infty)$ for

$$
1 \leqq p<\min \left(2,2 \beta^{-1}\right) \text {, }
$$

and $H_{v+r}\left(F_{r, \rho}\right) \in L_{q}(0, \infty)$ for $\max \left(2,2[2-\beta]^{-1}\right)<q<\infty$. Also

$$
t^{\rho} H_{v}(R f)=H_{v}(f)-\sum_{,=0}^{n} a_{r, p} H_{v+r}\left(F_{r, \rho}\right) t^{r},
$$

and $\left(1+t^{\rho / 2}\right) H_{\nu}(R f) \in L_{2}(0, \infty)$, so that $\Re(R) \subset H$.

Proof. Evidently each $F_{r, \rho}(z)$ is continuous except at $z=1$. From Schwartz's inequality,

$$
\left|\left(z^{2}-1\right)^{\frac{1}{1 \beta}} z^{v+r-\frac{1}{4}} F_{r, \rho}(z)\right|^{2} \leqq\|f\| \int_{0}^{1} y^{2 v+1}\left(1-y^{2}\right)^{2 n+\beta}\left(z^{2}-y^{2}\right)^{-2 n+2 r-2} d y .
$$

Hence $\left|F_{r, \rho}(z)\right|=O\left(z^{-1-n-\beta}\right)$ as $z \rightarrow \infty$. Since the case $n=\beta=0$ is excluded, $F_{r, p}(z) \in L_{p}(b, \infty)$ for any $b>1$ and $p \geqq 1$. For $z$ near 1 it is straightforward to obtain from the above integral the estimates:

$$
\begin{array}{rlrl}
F_{r, \rho}(z) & =O\left(|z-1|^{-\frac{1}{2} \beta}\right) & & (\beta>1), \\
& =O\left(|z-1|^{-\frac{1}{2}} \log |z-1|^{-1}\right) & (\beta=1), \\
& =O\left(|z-1|^{-\frac{1}{2}}\right) & & (0 \leqq \beta<1) .
\end{array}
$$

This shows that $F_{r, \rho}(z) \in L_{p}(0, \infty)$ for $1 \leqq p<\min \left(2,2 \beta^{-1}\right)$. The statement about the transform follows from Theorem 2 .

To prove $(A 4)$ it is first noted that

$$
\int_{0}^{1} f(y) d y \int_{0}^{1}(x t)^{\frac{1}{2}} J_{v}(x t) R(x, y) d x=\int_{0}^{1}(x t)^{\frac{1}{2}} J_{v}(x t) d x \int_{0}^{1} R(x, y) f(y) d y .
$$

For, $R(x, y) \geqq 0$, and $R$ is a bounded operator on $L_{2}(0,1)$. Thus by Schwartz's inequality and Fubini's theorem the order of integration may be changed. Hence, from $(A 3)$,

$$
\begin{aligned}
t^{\rho} H_{v}(R f) & =H_{v}(f) \\
& -\sum_{r=0}^{n} a_{r, \rho} t^{r+\frac{1}{2}} \int_{0}^{1} y^{v+\frac{1}{2}}\left(1-y^{2}\right)^{\frac{1}{2} \rho} f(y) d y \int_{1}^{\infty} \frac{z^{-v-r+1} J_{v+r}(t z)}{\left(z^{2}-y^{2}\right)^{n-r+1}\left(z^{2}-1\right)^{\frac{1}{2} \beta}} d z .
\end{aligned}
$$

Now it has been shown that $F_{r, \rho}(z) \in L_{1}(0, \infty)$, this being true if $f$ is replaced by $|f|$ in its definition. By Fubini's theorem the order of integration may be changed, and this gives $(A 4)$ immediately.

Since $R f \in L_{2}(0,1)$ then $H_{v}(R f) \in L_{2}(0, \infty)$, and so $t^{\rho / 2} H_{v}(R f)$ is square integrable on $[0,1]$. It only remains to consider large $t$. First take $\beta=0$. Then by $(A 2)$

$$
t^{n} H_{v}(R f)=t^{-n} H_{v}(f)-t^{-n+\frac{1}{2}} \sum_{r=0}^{n-1} b_{r} t^{r} J_{v+r}(t) \int_{0}^{1} y^{v+\frac{1}{2}}\left(1-y^{2}\right)^{r} f(y) d y .
$$


For large $t$ the right hand side is $O\left(t^{-1}\right)$. Hence $t^{\rho / 2} H_{v}(R f) \in L_{2}(\sigma, \infty)$. If now $\beta \neq 0$ then by $(A 4)$

$$
t^{\frac{1}{2} \rho} H_{v}(R f)=t^{-\frac{1}{2} \rho} H_{v}(f)-t^{-\frac{1}{2} \beta} F(t)
$$

say, where $F(t) \in L_{q}(0, \infty)$ for $\max \left[2,2(2-\beta)^{-1}\right]<q<\infty$. By Holder's inequality

$$
\int_{1}^{\infty}\left|F(t) t^{-\frac{1}{2} \beta}\right|^{2} d t \leqq\left\{\int_{1}^{\infty}|F(t)|^{q} d t\right\}^{\frac{2}{q}}\left\{\int_{1}^{\infty} t^{-\frac{\beta q}{q-2}} d t\right\}^{\frac{q-2}{q}} .
$$

The second integral is finite if $q>2,(\beta \geqq 1)$ or $q>2(1-\beta)^{-1},(0<\beta<1)$. These conditions are consistent with a choice of $q$ for which $F(t) \in L_{q}(0, \infty)$. This shows that $t^{-\beta / 2} F(t) \in L_{2}(0, \infty)$, and the concluding statements of the Lemma follow immediately.

Lemma A2. Suppose $f \in L_{2}(0,1)$ and for some positive integer $r$,

$$
t^{r} H_{v}(f) \in L_{2}(0, \infty) \text {. }
$$

Then there is a $g \in L_{2}(0,1)$ such that the following relation holds almost eveywhere:

$$
t^{r} H_{v}(f)=H_{v+r}(g) \text {. }
$$

Proof. First suppose $r=1$. Since $t H_{v}(f) \in L_{2}(0, \infty)$ there is evidently no loss of generality in taking $f$ to be continuous. It follows that $f(1)=0$. Now set

$$
g_{B}(u)=\int_{0}^{B} t(t u)^{\frac{1}{2}} J_{v+1}(t u) H_{v}(f) d t .
$$

As $t H_{v}(f) \in L_{2}(0, \infty)$, then as $B \rightarrow \infty, g_{B}(u)$ must tend in $L_{2}(0, \infty)$ to a limit, say $g(u)$, with $t H_{v}(f)=H_{v+1}(g)$ p.p. By Schwartz's inequality,

$$
\lim _{B \rightarrow \infty} \int_{x}^{1} u^{-v-\frac{1}{2}} g_{B}(u) d u=\int_{x}^{1} u^{-v-\frac{1}{2}} g(u) d u \quad(x>0) .
$$

Substitution for $g_{B}(u)$ from $(A 7)$ gives after a change of order of integration, valid by Fubini's theorem,

$$
x^{\nu+\frac{1}{2}} \int_{x}^{1} u^{-v-\frac{1}{2}} g_{B}(u) d u=\int_{0}^{B}(x t)^{\frac{1}{2}} H_{v}(f)\left[J_{v}(t x)-x^{v} J_{v}(t)\right] d t
$$

Let $B \rightarrow \infty$. Then by $(A 8)$ since $f(1)=0$,

$$
f(x)=x^{v+\frac{1}{2}} \int_{x}^{1} u^{-v-\frac{1}{2}} g(u) d u \quad(x>0) .
$$

As $f(x)=0$ for $x \geqq 1$ then $g(u)=0$ p.p. for $u>1$. The proof is complete for $r=1$, and the result for general $r$ follows by a repetition of the argument.

It was proved in Lemma $A 1$ that $\mathfrak{R}(R) \subset H$. The proof of the theorem will be complete if it can be shown that $(A 1)$ holds. Suppose first that $\beta \neq 0$. From Lemma A2

$$
t^{n} H_{v}(g)=t^{n-r} H_{v+r}\left(g_{r}\right)
$$


for some $g_{r}$ in $L_{2}(0,1)$. By an application of Holder's inequality it is easily seen that $H_{v+r}\left(g_{r}\right) \in L_{p}(0, \infty)$ for $2(1+\beta)^{-1}<p<2$. Hence from $(A 4)$, for all $f \in L_{2}(0,1)$ and $g \in H$,

$$
\left(t^{\frac{1}{2} \rho} H_{v}(R f), t^{\frac{1}{2} \rho} H_{v}(g)\right)=\left(H_{v}(f), H_{v}(g)\right)-\sum_{r=0}^{n} a_{r, \rho}\left(H_{v+r}\left(F_{r, \rho}\right), H_{v+r}\left(g_{r}\right)\right),
$$

where $F_{r, \rho} \in L_{p}(0, \infty)$ for $1<p<\min \left(2,2 \beta^{-1}\right)$. It is clearly possible to choose $p$ so that both $F_{r, \rho}$ and $H_{v+r}\left(g_{r}\right) \in L_{p}(0, \infty)$. Since $F_{r, \rho}$ and $g_{r}$ have supports in intervals with intersection of measure zero, by Theorem 2 each term in the summation vanishes and $(A 1)$ is proved. Finally, if $\beta=0$, the above proof will hold except for the term $r=n$. In this case both $g_{n}$ and $F_{n, 2 n} \in L_{2}(0, \infty)$. The result now follows from Theorem 1 . This concludes the proof of Theorem A1.

\section{REFERENCES}

(1) I. W. BUSBRIDGE, A theory of general transforms for functions of the class $L_{p}(0, \infty),(1<p \leqq 2)$, Quart.J. Math. Oxford Ser. 9 (1938), 148-160.

(2) J. C. COOKe, A solution of Tranter's dual integral equations problem, Quart. J. Mech. Appl. Math. 9 (1956), 103-110.

(3) A. ERdelyi and I. SNeddon, Fractional integration and dual integral equations, Canad. J. Math. 14 (1962), 685-693.

(4) V. Hutson, The asymptotic solution of a class of integral equations, J. Math. Anal. Appl. 20 (1967), 380-396.

(5) V. Hutson, The circular plate condenser at small separations, Proc. Cambridge Phil. Soc. 59 (1963), 211-224. 1966).

(6) T. Kato, Perturbation Theory for Linear Operators (Springer Verlag, Berlin,

(7) E. R. Love, The electrostatic field of two equal circular coaxial conducting disks, Quart. J. Mech. Appl. Math. 2 (1949), 428-451.

(8) R. K. Mahalanabis, A mixed boundary value problem of thermoelasticity for a half-space, Quart. J. Mech. Appl. Math. 20 (1967), 127-134.

(9) I. SNeddon, Mixed Boundary Value Problems in Potential Theory (Wiley, New York, 1966).

(10) H. WIDOM, Extreme eigenvalues of $N$-dimensional convolution operators, Trans. Amer. Math. Soc. 106 (1963), 391-414.

(11) H. Widom, Extreme eigenvalues of translation kernels, Trans. Amer. Math. Soc. 100 (1961), 252-262.

\section{Department of ApPlied Mathematics \\ THE UNIVERSITY \\ SHEFFIELD, 10}

